\title{
Augmented reality an economical solution for engineers and designers
}

\author{
Sidra Nasir ${ }^{1}$, Mohammad Noman Zahid ${ }^{2}$, Talha Ahmed Khan ${ }^{3}$, Kushsairy Kadir ${ }^{4}$, Sheroz Khan $^{5}$ \\ ${ }^{1,2}$ Faculty Of Science and Technology, Ilma University Formerly IBT, Pakistan \\ ${ }^{3}$ Universiti Kuala Lumpur, British Malaysian Institute (BMI), Malaysia \\ ${ }^{3}$ Usman Institute of Technology (NED University), Pakistan \\ ${ }^{4}$ Universiti Kuala Lumpur, IPS, UniKL1016, Malaysia \\ ${ }^{5}$ Department. of ECE, Faculty of Eng. International Islamic University (IIUM), Malaysia
}

\section{Article Info}

Article history:

Received Jun 5, 2019

Revised Aug 7, 2019

Accepted Aug 21, 2019

\section{Keywords:}

$3 \mathrm{D}$ visualizer

Architects

Augmented reality

Interior designers

Workspace

\begin{abstract}
Now a days, with obscene workload and a busy life, many experts face problems which more or less result in loss of their customers or to certain overheads that immobilize the customer's satisfaction process. The software being proposed will facilitates professional from various field like in mechanical designing, interior designing and that of architects. This research proposal most likely acts as an effective tool that can reduce the gap between industrial enterprises and customers in addition to other relevant business groups. It helps to visualize architectural designs and interior designs. Before the physical implementation, a virtual model of a real environment can be designed, enabling designers to virtually implement their idea in the given workspace and then view it in a real environment, enabling designers to view their $3 \mathrm{D}$ visualizations on their $2 \mathrm{D}$ drawings this help them to initially visualize and reconstruct the design without wasting a large amount of money making it a cost-effective solution. Application is based on my observations of the user's aspirations of an augmented reality in design service, a service that combines different social media functionality, augmented reality (AR) and 3-D modeling that embraces the concept of home design, architecture and mechanical designing process. This study connects all users of relevant concerns to an augmented reality's userintensive design. The paper provides you with the existential complexity of how the AR can be implemented to enhance the work of architects and designers. The proposed application shows two types of reality, marker-less and more marker-based. The new feature of the project is that no expensive devices are required. It can be used easily through smartphones and tablets. This is a cost-effective solution that mainly reduces the overhead accosted daily by, mechanical designers, interior designers and architects. The software displays all the features that mechanical designers, interior designers and architects may need.
\end{abstract}

Copyright (c) 2020 Institute of Advanced Engineering and Science. All rights reserved.

Corresponding Author:

Kushsairy Kadir,

Department of Electrical and Electronic Engineering,

British Malaysian Institute, Universiti Kuala Lumpur,

Batu 8, Jalan Sungai Pusu, 53100, Selangor, Malaysia.

Email: kushsairy@unikl.edu.my

\section{INTRODUCTION}

It is a difficult challenge for anyone to understand how a certain object will look in the real world before sampling and production is carried out. This is a current technology which incorporates the placement of certain virtual objects in the real environment. This reality is also referred to in the general context as a 
mixed reality that deals with a multitude of domains that involves virtual reality (VR) as shown in Figure 1. This technology has been used in different fields such as in reparations, medicine, tele-robotics, manufacturing, robotics, maintenance, engineering and education.

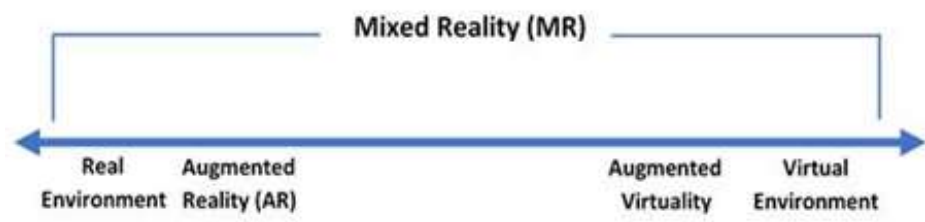

Figure 1. Paul Milgram's point of view about augmented reality [1]

The real-virtual continuum of Paul Milgram's Augmented systems of reality integrate the virtual world and the real world so that the end user experience them together. AR's key advantage is to find digital tools in the precise region and position, making the monitoring tool one of the most important features of an AR system. An AR system should basically be able to dynamically comply with the user's perspective and retain virtual elements aligned with the actual environmental entity. A camera for image capture and computer hooked-up application software are the principal attributes in an AR system, so are various hardware forms.

\section{RECENT TRENDS}

Augmented reality helps you in solving real lifestyle problems. Issues are based mainly on elements such as money and time. The trouble has now become the overhead in industrial designing. In head-mounted displays and monitors, hardware implementation is specifically designed for expert users [2]. Android application for Augmented Reality was the key to solving these problems, given that it is an Android application, supported by android devices such as smartphones that are easily accessible to end-users. The research on applications of the Augmented Reality within the architectural discipline and its usefulness in promoting precision in decision-making [3]. It is difficult to imagine how any object appears in a room or in an open area with many aspects left to the imagination of the end user. The scale, color and how it is integrated in the existing environment. Increased reality, the technology that places computer graphics in the real environment has several applications in the architecture and engineering discipline and has been used to address certain real-world issues. Previously it was referred to as mediated reality, in which a view of reality is altered through a computer; the technology has evolved one's notion of reality. Head-mounted display (HMD), contact lenses, eye lenses and monitors are some of the accessories that have integrated increased reality. Common people or non-professionals can also take advantage of the augmented reality to solve their real problems [4].

The wearable display offers great experiences in relation to augmented reality with a non-invasive and wide monitor. Such wearables, however, it is costly and everyone doesn't have sufficient funds to own it. In addition, this device is constructed with many sensors for gaming applications to improve its user experience. The system becomes more complex and costlier for the app design. So, it's not always affordable [5]. This Augmented System allows end users to estimate the length of the space captured by the sensor. It also provides end-user with an interface to select the object and manipulate it. A priority strategy is adopted to prioritize automatically the size of the furniture according to the size of the room [6]. A system has been programmed to automatically arrange furniture. They used and enforced spatial and functional links on existing computer vision. It was based on an innovative application which would help end-users to decorate their homes. This snatches the opportunity from users to use this application to implement their own ideas [7]. The Augmented reality has become a medium for the purchase of relevant furniture. Markers are placed in the required area (e.g. floor or wall) to define the scale and the room coordinates. The end user selects the virtual object and pushes it to the increased area. The idea used here to combine the selected virtual furniture in the environment in which the actual furniture already exists. All this is done in order to conveniently buy the furniture, saving resources and effort by avoiding the need to go to the store and select the furniture [8]. It is difficult to placate the customer's satisfaction when you buy the furniture for your room without an imaginary vision. In this way, the user can only drag an object into the design space. And let the user see how they look in their real environment. A marker-based application can be found in the archive containing the textual data, allowing the layman to experience an augmented reality based on Marker. 
Today everyone is busy with their work, so they do not have the time to visit many shops to buy furniture for their regular use. It nevertheless led to a reduction in end-user expenditure [9]. This report shares about the research and development of applications in design and manufacturing for increased reality (AR) applications [10]. This article discusses the problems concerning the future of education in interior design. Those problems could be the basis for concepts, technology integration, etc [11]. This study discusses how distributed AR applications can be designed to facilitate applications in interior design [12]. Virtual furnishings and changes to a new strategy for interior design training through augmented reality technology are being observed in this research [13]. This paper addresses users ' complications when tracking a virtual object on the top of the table [14]. This issue discusses a car design process, one of the main issues is that the virtual content is projected onto the real object with very high visual quality, because decisions are taken based on this projection design. Approach to that increases the visual quality of the images that can comply with the design process requirements [15].

This article discusses new method for the augmentation of real objects capable of satisfying the design process requirements. We are presenting a new ray tracing rendering method that increases the visual quality of images compared to existing methods. The values desired for these images need to be further adapted to the material, environmental light and local orientation of the projector. To achieve it, physically based calculation that precisely determines the correlating projection elevations for these values using threedimensional search tables on each projection pixel are developed [16]. This paper reviews Augmented Reality (AR) research and development of applications in design and production processes [17]. For the use of augmented-spatial reality in automotive processes and applications, an important problem is that the virtual content must be projected onto a real object with very high accuracy, as decisions are made based on projection. This article therefore presents a new method for the evaluation of geometric recording methods that a projector aligns with a real object [18]. This paper aims to provide a practical tool for analyzing the various elements and constraints of current neuro-navigation technologies and encouraging the creation of new neuro-chirurgical AR alternatives [19]. This article intends to demonstrate the present state of the art of AR in maintenance and the most appropriate technical constraints through the outcomes of a systematic literature assessment [20]. In this study, we aim to explain and learn to what extent mobile AR applications contribute to intelligent retail environments by generating added value for clients and retailers [21]. This article intends to present an almost self-contented introduction to the most significant vision-based camera location methods along with a study of several extensions suggested in latest years [22].

A tracking technique based on the mixture of point cloud and visual function is suggested to enhance the applicability and robustness of the three-dimensional tracking technique of an augmented realityaided assembly guidance system for mechanical products [23]. Another article by Jain, P., Manweiler, J. and Roy Choudhury, R. also discusses, what is needed to allow augmented reality (AR) on smartphone [24]. The proposed augmented reality system CMARTS effort to investigate the merging of enhanced reality and integrated training skills into deployed hand-held mining detectors to promote performance training at home and in operational settings [25]. This research article proposes a fresh method that addresses the challenge of experimentation in the provision of distance learning. It utilizes an adequate haptic glove with internet wearable technology [26].

Table 1. Data Collection and Comparative Results

\begin{tabular}{lllc}
\hline \multicolumn{1}{c}{ Method } & \multicolumn{1}{c}{ Merit } & \multicolumn{1}{c}{ Challenges } & Resources Spent \\
\hline Questionnaire and Surveys & $\begin{array}{l}\text { Inexpensive to manage, } \\
\text { Easy to analyze can be adapted into many } \\
\text { forms }\end{array}$ & $\begin{array}{l}\text { Might not get careful answers. } \\
\text { Answers might be biased. }\end{array}$ & Low \\
Interviews & $\begin{array}{l}\text { Eliminates the gap of understanding } \\
\text { between stakeholders. }\end{array}$ & $\begin{array}{l}\text { Can be hard to analyze. } \\
\text { Might take a lot of time }\end{array}$ & High \\
Case Studies & $\begin{array}{l}\text { Powerful mean of strategical information. } \\
\text { Wocument reviews }\end{array}$ & $\begin{array}{l}\text { Till help Consuming obtaining Inclusive past } \\
\text { Data is restricted to what } \\
\text { information. }\end{array}$ & High \\
\hline
\end{tabular}

Table 1 shows the approaches pursued above and also their advantages and disadvantages. The second challenge is data collection, because in many countries including Pakistan, reality is not widely known. People don't know how it works, but we got some answers and saved the various reactions from the architects, car designers and the interior designer. Determine features and discuss their technical and practical feasibility of the AR interior design service for various target groups A scenario-based survey, co-design sessions studied user expectations for AR interior, car design and architecture design services Focused interviews and sessions. In total, the survey responded to the visual design service by 100 consumers and professionals. Special co-creation meetings were held afterwards with five consumers, two interior designers 
and three architects to get to know the individual groups. Furthermore, we interviewed a number of different trade users in the field to learn more about product expectations. The combination of various user-centered approaches proved to be a valuable input in the main concept design stages.

Following the interview and the group discussions with the specific domain staff, this easy to use design tool came to the conclusion that they need this type of an app. It reduces their overhead, and also makes them understand the idea behind the design in the primary stage by providing 3D visual support, as previously discussed. It appears that there is a demand both on consumers and professionals for user-friendly design tools.

\section{METHODOLOGY}

The influence of print media (images, panaflex, print outs) is incapable and sufficient to satisfy designers and architects to visualize their ideas and complicated things in the real world in their desired way. The application converts 2D maps to 3D by scanning (especially for architects) and end users can keep their schedules and set the reminder, share their work with the community (blog) and understand other designers ' work that will give them new motivation. Can also upload their on custom model. This application can only be accessed by authorized users (as we have registered and signed up). The user is able to interact in real time with virtual 3D models of furnishings through an augmented reality and change the colors of furnishings and walls or furniture. This enables the exploration and visualisation of complex and innovative designs, which make AR technology accessible to both pros and amateurs to interior designers and architects. Two types of augmented reality are incorporated and associated to a particular discipline in the applications. Aim of an application is to reduce the overhead of their paper work and the expenses they consume in dealing with the ambiguity of their clients, and this is the complete package for designers and architects. This will also help you maintain your schedule while promoting your work to be recognized. This application also offers new skills from qualified veterans for beginners. This application also offers to learn from qualified veterans. It also helps architests and designers learn important things that are often learned through trial and error. In the past, the applications were focused only on the provision of home furnishing by promoting its products. The two distinct types of AR on one common ground have not been yet.

\subsection{Module for Architecture}

Marker based augmented reality is used to enable the functionality developed for architects. A marker is required to view the rendered 3D object. An image or a representation that can be perceived by an image tracking module using methods consisting of pattern recognition, image processing and computer vision techniques can be defines as a marker. It defines the correct size and position of the camera once detected. In the process of marker detection, after deciphering its identity and approved as a marker. Using this feedback, the application calculates the position of the 2D image. The tracking module comprises of two main phases. First, the application detects the marker and evaluates the co-ordinates of the marker. Hence, implements it on the available space. Then, the tool will provide an automatic arrangement of the building according to the 2D image. Use either SI (MKS) or CGS as primary units. (SI units are encouraged.) English units. At last, a proposed display plan of the building will be rendered into the real scene. It is the heart of one part of an application. This module starts when the AR camera is launched. After the calibration of the camera and it gives feedback to the AR camera in real time. The camera evaluates every image in the scene and detects the markers. The marker coordinates are tracked and marker's position are calculated and sent to the AR camera. The coordinates are sent to the Image rendering module where an object is rendered on it.

\subsection{Module for Interior Designing}

Marker-less AR is another part in which location and mapping is simultaneously done that allows you to place your AR experience pretty much wherever you want. This technique is the heart of another part of an application, specifically designed for interior designing. In this Module, Camera calibrates and the coordinates of the area is calculated. An object appears when an end-user selects an object. When the end-user desires to change the objects pose and size. Their fingers gesture on the selected object is analyzed if the gestures are identified then the camera is calibrated and when the pose change is approved then it is displayed in real time. This allows the users to interact with multiple virtual object in real-time and later can capture its image for safe keeping.

\subsection{Module for Mechanical Designing}

Here in this module, there are various aspects, geometric simulator, 3d Generator, end-user is allowed to design their product and study its physical and behavioral to overcome design challenges and product engineering. The user has been provided with tool that will help them in drawing and it will later on 
transits into a $3 \mathrm{~d}$ project and this functionality can be achieved while user is drawing the co-ordinates are placed on them and the object can be manipulated by it when its mode is switched on. User is presented with an interface where they can sketch manipulating view using viewcube. Using the drawing tool straight line or curve can be drawn, made by clicking the tool to create two anchor points. Position the tool where you want the curve to begin, and hold anchor point appears, drag to set the slope of the curve segment you're creating and can also add anchor point on need. While drawing width height and depth shall appear in slelected unit, notifing the end user to keem measurments in check. 3D drawiing can be achieved by manipulating the cube box that is interleaved with the drawing plane by clicking on 3D button the sketch solidifies transists into $3 \mathrm{~d}$ mode that is faces and edges are created. After the conversion the designer can still manipulates the edges, faces and co-ordinates(vertices) to make adjustments to design to avoid overheads spent on clay modeling or 3D printing. Designers can also assign materials and colors to the $3 \mathrm{~d}$ objects to give it a realistic look. Eraser has been provides to erase any unnecessary anchor points. End user can also design the internal edifice. For Further detailig, in design more functionalities has been provides like extrude or vice versa, bevel on selection of the face or vertices, edges in 3D mode. To enhance the usability of designing end-user can switch to Augmented reality AR mode to further view their design in real environment.

\subsection{Module for Camera Tracking}

If Calibration about the stationary details of a scene then the system has to deal with dynamic scene changes too. Therefore, it estimates the scene for instance, certain features especially in interior design module. As for the Module dedicated to Architects on detection of tracking point on the 2D image it calibrates itself.

\subsection{Algorithms}

Image Tracking Module 1 as shown in Figure 2.

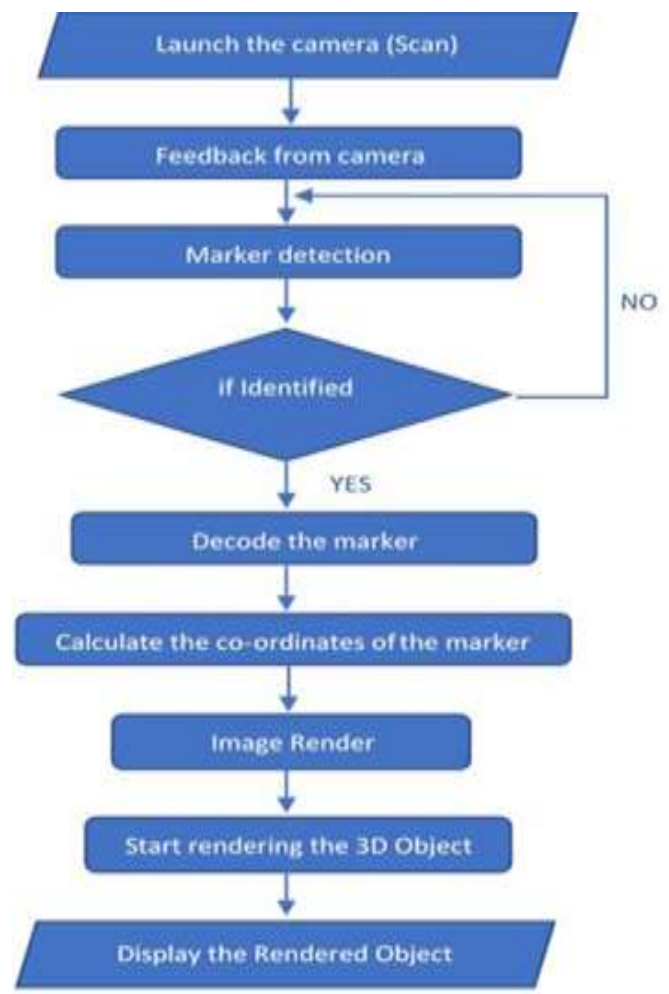

Figure 2. Image tracking module 1

\section{Image Tracking:}

Create class (Image Tracking)

Create variables (Set the below variables $=0$ )

a) Camera Position

b) Camera Angle 
c) Camera Target position

d) Camera Target Angle

Launch and calibrate Camera

If (Image $=$ Marker)

Start Rendering the 3D Object

If (Image (Marker) Moves)

Move the rendered model with it

Send information to Camera tracking Module

If (Marker is Lost)

Disable Image Rendering

Else Do Nothing

Object Tracking/Rendering Module as shown in Figure 3.

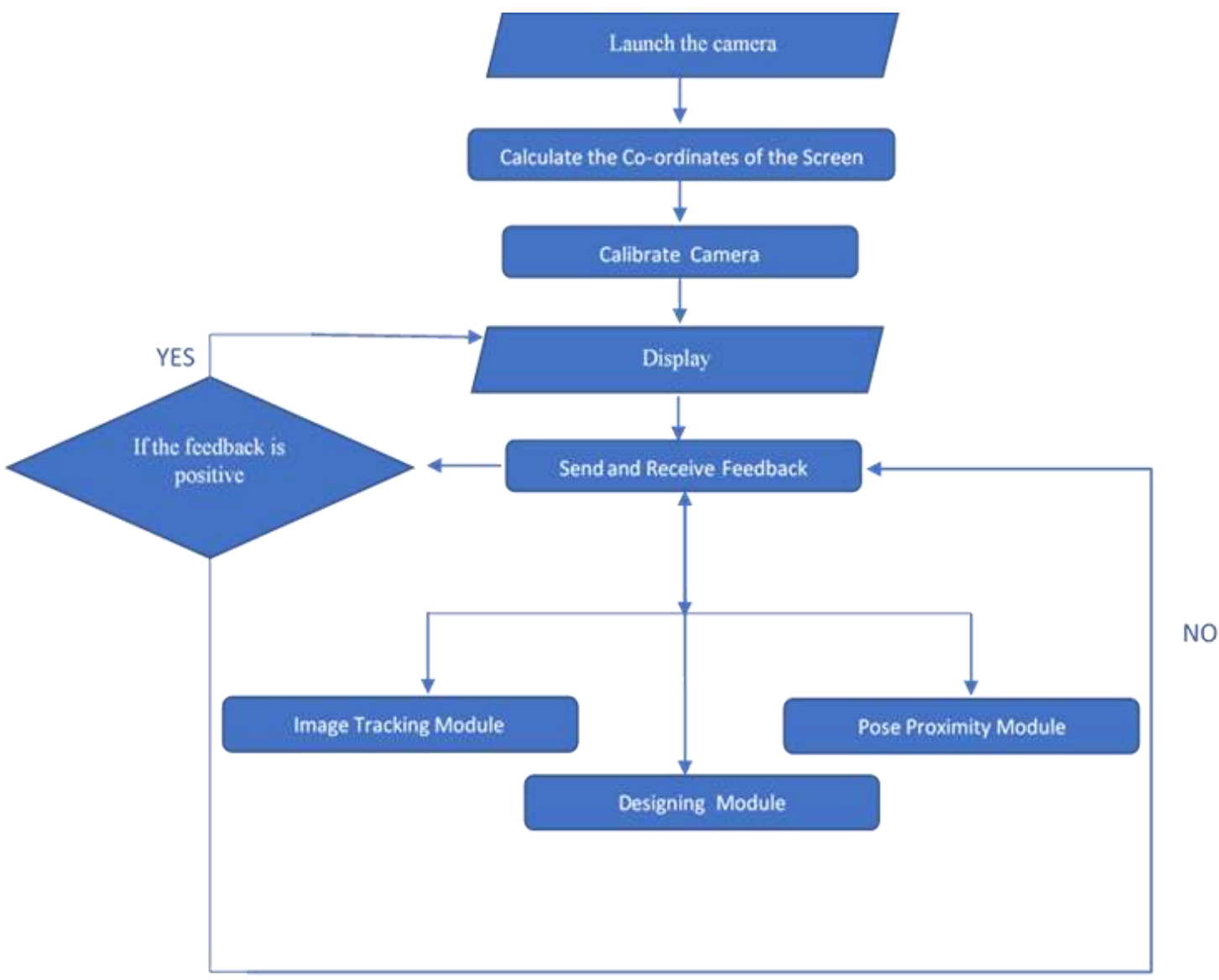

Figure 3. Object tracking/rendering module

\section{Object Tracking/Rendering Module:}

Create class (Pose and Proximity detection (Object Tracking))

Create variables (Set the below variables $=0$ )
a) Camera Position
b) Camera Angle
c) Camera Target position
d) Camera Target Angle
e) Object default Position
f) Object default Angle
g) Object User-defined Position
h) Object User-defined Angle
Launch and Calibrate Camera 
Estimate the real environments (magnitude and co-ordinates)

If (On-Click Button (Object))

Load the object in default pose

If (On- Click Object (Select))

Enable Scale, Rotate, Move

If $($ User Input $($ Motion $)=$ Move $)$

Move the object

Send information to Camera tracking Module

If (User Input (Motion) $=$ Scale)

Resize the object

Send information to Camera tracking Module

If $($ User Input $($ Motion $)=$ Rotate $)$

Rotate the object

Send information to Camera tracking Module

Else make no Change

Camera positioning and tracking module as shown in Figure 4.

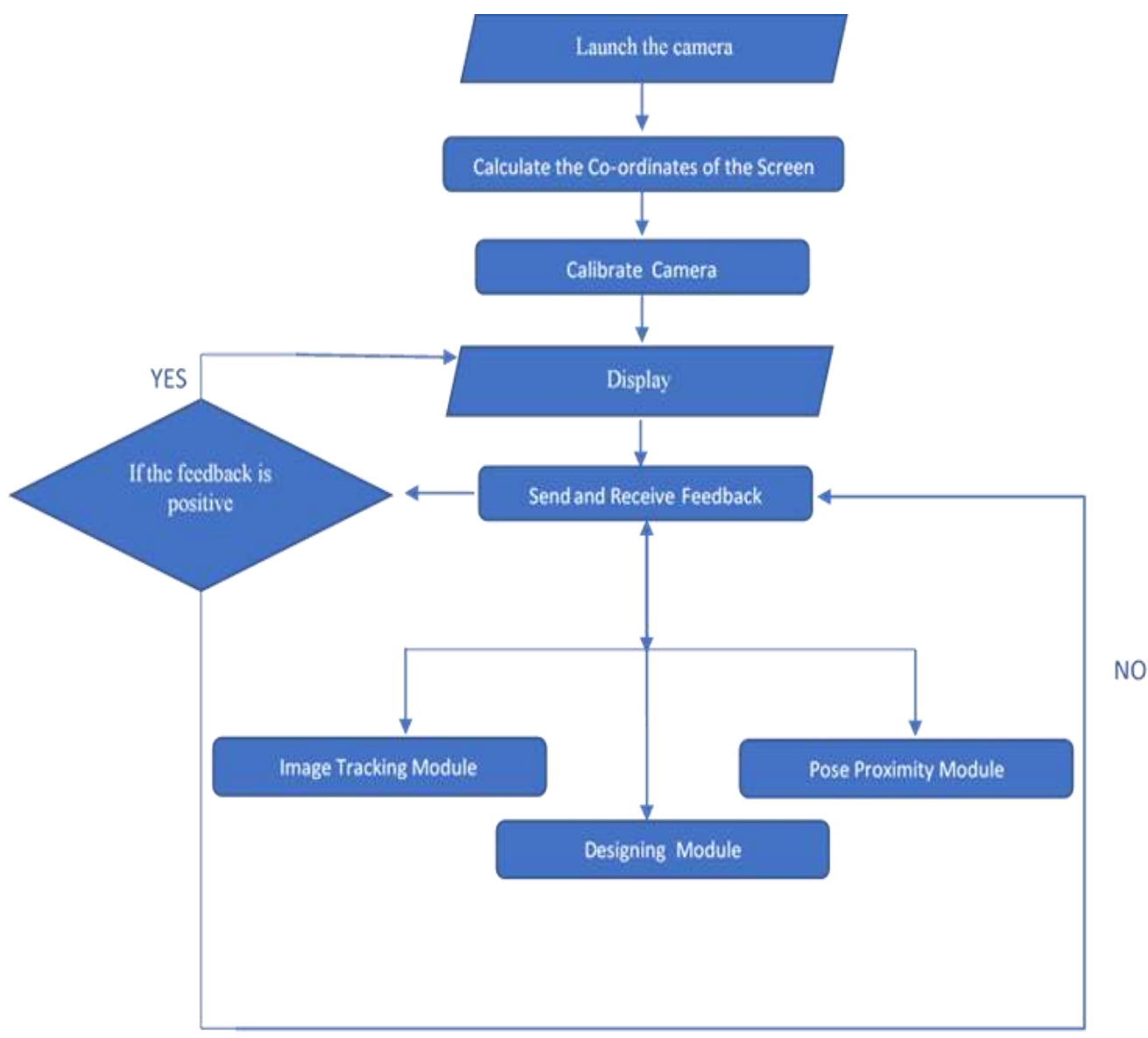

Figure 4. Camera positioning and tracking module

\section{Camera Tracking:}

Create class (Camera Tracking)

Create variables (Set the below variables $=0$ )

a) Camera Position

b) Camera Angle

c) Camera Target position

d) Camera Target Angle

On start (Launch and Calibrate Camera) 
Send Feedback to Image Tracking Module

Send Feedback to Object Tracking Module

If (Image $=$ Marker)

Estimate the Markers Position

Start Rendering the 3D Object

If (Feedback from image tracking module

(verifies the marker) $=$ true)

Start Displaying the rendered Object

If (Feedback from object tracking module $($ Motion $)=$ true $)$

Update the display of an Object

Else Do Nothing

Mechanical design module as shown in Figure 5.

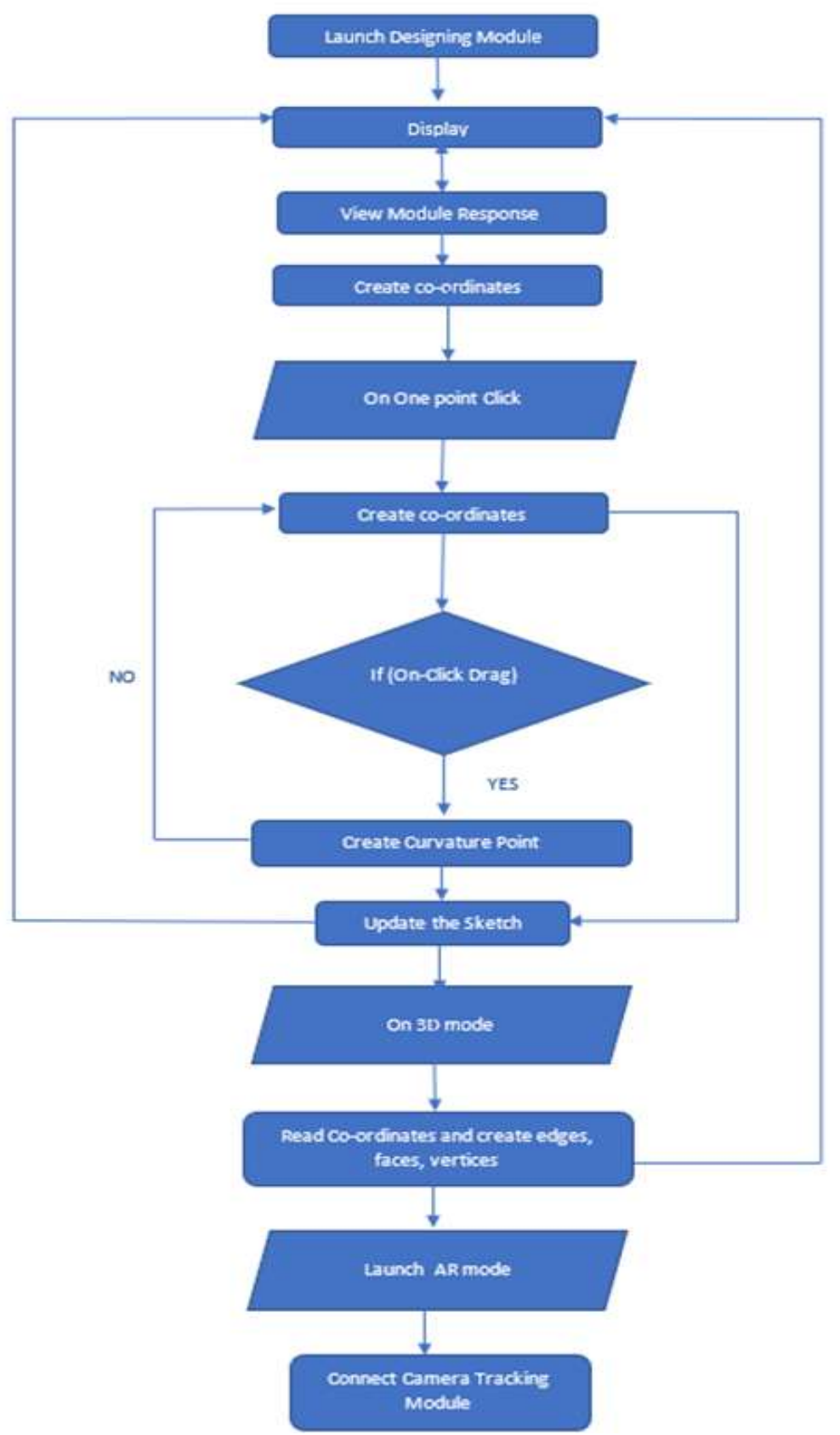

Figure 5. Mechanical design module 
Mechanical Designing:

Create class (Mechanical Design)

Create variables (Set the below variables $=0$ )

a) Sketch $=$ user input

b) Color

c) Shape

d) Eraser

e) Material

f) Curvature $=$ user input

Launch and Calibrate View Plane (Default Orthographic)

Create Sub Class Display

If $($ User Input $($ Motion $)=$ Zoom In $)$

Apply Zoom In

If $($ User Input $($ Motion $)=$ Zoom Out $)$

Apply Zoom In

If (On- One Click in Sketch Mode)

Create co-ordinate

If (On- Click Drag in Sketch Mode)

Create Curvature

Update Display

On User Input of view plane

If (On-Click Button (Perspective))

Disable Orthographic views

If (On-Click Button (Top))

Enable Orthographic views

Disable Perspective view

If (On-Click Button (Left))

Enable Orthographic views

Disable Perspective view

If (On-Click Button (Right))

Enable Orthographic views

Disable Perspective view

If (On-Click Button (Bottom))

Enable Orthographic views

Disable Perspective view

Update View PlaneUpdate Display

If (On-Click Button (3D Mode))

Estimate the co-ordinates (magnitude and co-ordinates)

Create Object Vertices, edges, face - On Solidification

On user Assign Material

If user Input= Material type (Chrome, Blinn, Mirror, Transparent, Plastic (fiber))

Apply Material type

If AR Mode Activated

Connect Camera Tracking Module

\section{RESULT AND DISCUSSION}

This application is relatively new, this application allows the user to add multiple object to the screen in real-time. The user can manipulate the object like, move, rotate, resize and change the color or texture of the object on selection. Thus, allowing the interior designer to implement their design and evaluate them in real- environment. In this application one module is endowed to Architectures where over their 2D image a $3 \mathrm{~d}$ object is rendered to let them consider and assess their idea.

Figure 6 show the home Screen of the Application. Here user will sign up create his profile. In Case anyone who have no account on application can excess the restricted version using skip button. Figure 7 show the help panel, where the user is who is using the application for the first time can be guided. Figure 8 show the Blog / Community Page. Here, user can share their work and converse with other users and learn new things. Figure 9 show the Calendar/ Scheduler Panel. Here, the user can schedule his meeting. After clicking any date in the calendar, user will be directed to the reminder panel. He can write his notes as well as set the reminder for meeting, which he will later receive via notification on selected date. Figure 10 
show the Create Room Panel and one part of the application. This panel enables the user to implement their design by dragging the object on to the Screen. This Panel shows all the functionalities of Augmented reality (Marker-less). It also exhibits the Categories that is provided to the user to use. Figure 11 reveals that on selection of the object the texture and delete button will be visible. Figure 12 show the Panel dedicated to Architects. This is known as the Architecture Panel and another major part of the application. This panel scans Image containing the tracking points which is later on scanned and when authorized over the 2D image target, rendering of the 3D models commences. Figure 13 is concerned with mechanical designing, here the user can sketch and solidifies the mesh and can also view their design in AR mode to further analyze their model. Figure 14 reveals the front and sideview plane that can be manipulate while designing. Figure 15 portrays the designed model in augmented reality mode when vertices and everything is still present and can be manipulated while still in the real environment.

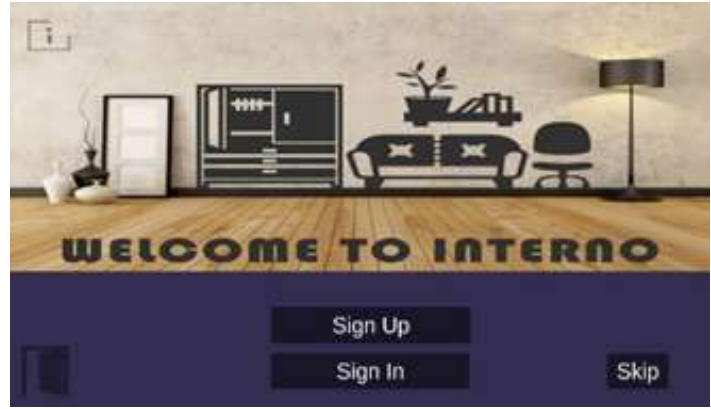

Figure 6. Home screen of the proposed application

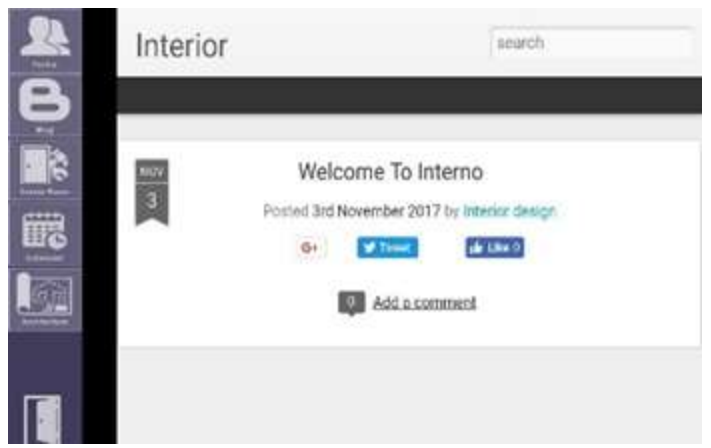

Figure 8. Community page

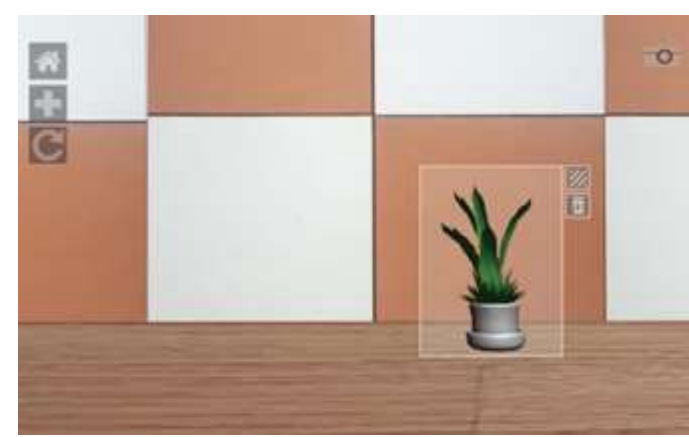

Figure 10. Create room panel

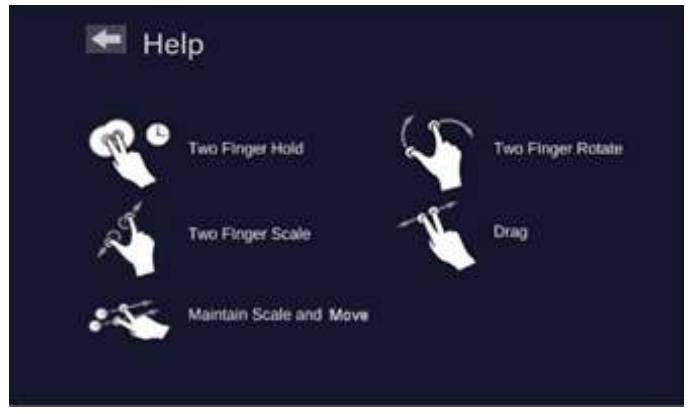

Figure 7. Help panel

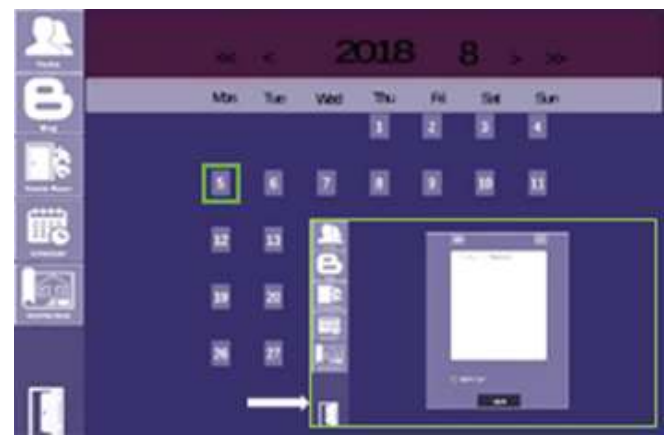

Figure 9. Scheduler \& reminder panel

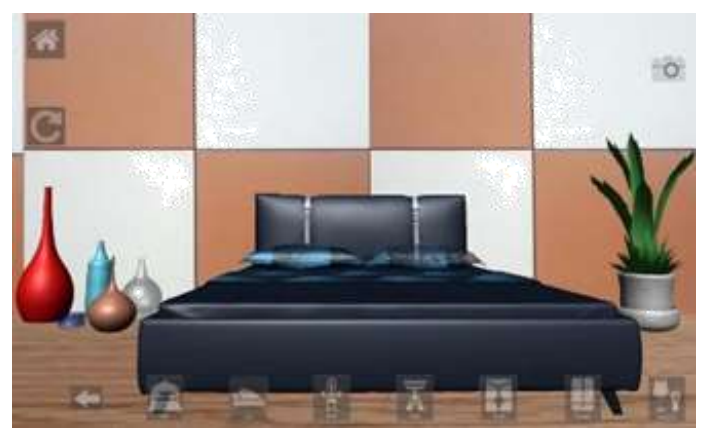

Figure 11. Create room panel with object editing panel 


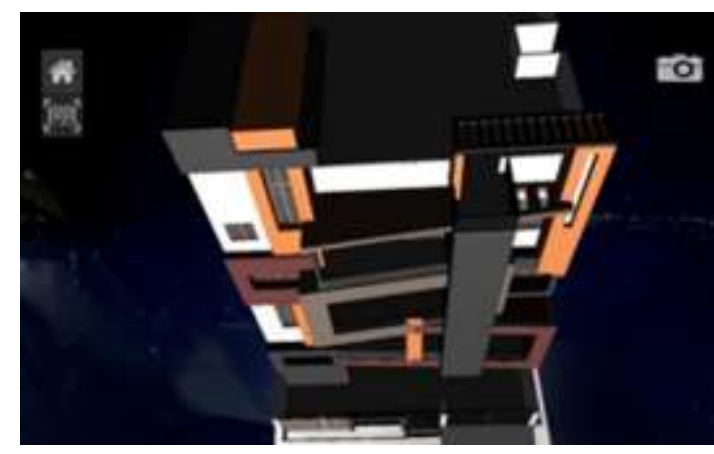

Figure 12. Architect panel
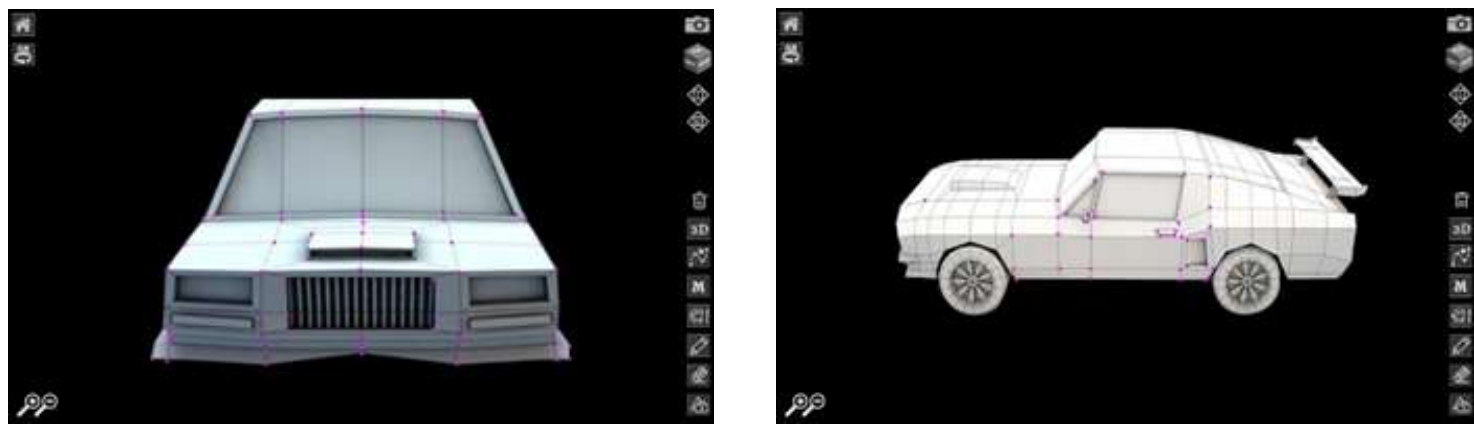

Figure 14. Mechanical design panel (front and side view)

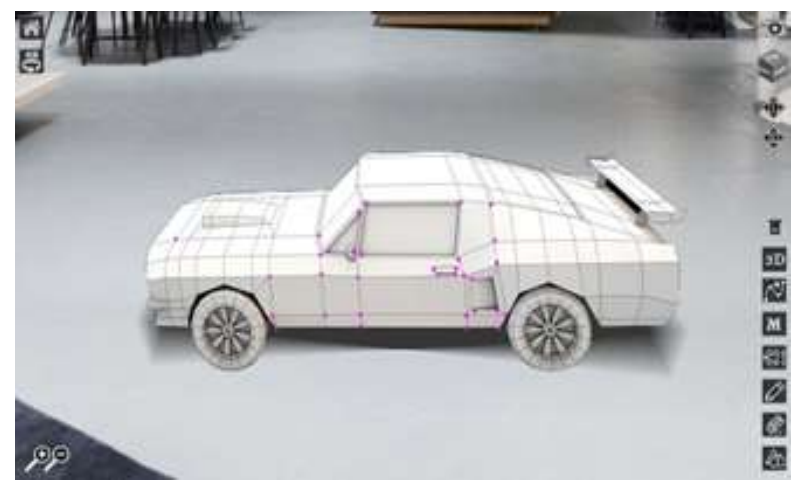

Figure 15. Mechanical design with the control functions in AR mode

\section{CONCLUSION AND FUTURE ENHANCEMENTS}

The proposed solution is based on the research regarding to its subject so we have found no applications like ours introduced yet. It will be very useful especially where client's minds change within a blink of an eye. We want to conclude our report that our research is a step to the technology advancement in the concerned fields, by implementing this research, we believed that their work flow pipeline will be benefited from it. In future, this application may acquire functionalities, like camera zoom in and out, the latest module can be indorsed by the addition of extensive animation and simulation.

\section{REFERENCES}

[1] P. Milgram, H. Takemura, A. Utsumi, and F. Kishino, “,” Telemanipulator and Telepresence Technologies,

[2] Klein and D. Murray, "Parallel Tracking and Mapping on a camera phone," 2009 8th IEEE International Symposium on Mixed and Augmented Reality, pp. 83-86, 2009.

[3] Nee, S. Ong, G. Chryssolouris, and D. Mourtzis, "Augmented reality applications in design and manufacturing," CIRP Annals, vol. 61, no. 2, pp. 657-679, 2012. 
[4] Prof. B.Y.Jani, Pratiksha Dahale, Ankita Nagane, Bhavika Sathe, and Nilam Wadghule, "Interior Design in Augmented Reality Environment," International Journal of Advanced Research in Computer and Communication Engineering, vol. 4, no. 3, pp. 286-288, Mar. 2015.

[5] William A.S. Buxton, "Living in augmented reality: Ubiquitous media and reactive environments," Video Mediated Communication. Hillsdale, N.J.: Erlbaum, pp. 215-229, 1997.

[6] J. K. Tang, et al., "AR interior designer: Automatic furniture arrangement using spatial and functional relationships," 2014 International Conference on Virtual Systems \& Multimedia (VSMM), pp. 345-352, 2014.

[7] R. T. Azuma, "The Challenge of Making Augmented Reality Work Outdoors," Mixed Reality, pp. 379-390, 1999.

[8] Matsuoka, A. Onozawa, and E. Hosoya, "Environment mapping for objects in the real world: A trial using ARToolKit," The First IEEE International Workshop Augmented Reality Toolkit, 2002.

[9] Khushal Khairnar, et al., "Furniture Layout Application Based on Marker Detection and Using Augmented Reality," International Research Journal of Engineering and Technology (IRJET), vol. 2, no. 7, pp. 540-544, 2015.

[10] Paul Merrell, Eric Schkufza, Maneesh Agrawala, Vladlen Koltun, "Interactive furniture layout using interior design guidelines," ACM Transactions on Graphics, vol. 30, article. 87, pp. 87:1- 87:9, Jul. 2011.

[11] Denise A., Guerin Ph.D., "Issues Facing Interior Design Education in the Twenty-First Century," Journal of interior Design, vol. 17, no. 2, pp. 9- 16, Sep. 1991.

[12] Klaus H. Ahlers, André Kramer, David E. Breen, Pierre-Yves Chevalier, Chris Crampton, Eric Rose, Mihran Tuceryan, Ross T. Whitaker, Douglas Greer, "Distributed Augmented Reality for Collaborative Design Applications," Computer Graphics Forum, vol. 14, no. 3, pp. 3 - 14, Aug. 1995.

[13] Viet Toan Phan, Seung Yeon Choo, "Interior Design in Augmented Reality Environment," International Journal of Computer Applications, vol. 5, pp. 16 - 21, Aug. 2010.

[14] Kato, M., et al., "Virtual object manipulation on a table-top AR environment," Proceedings IEEE and ACM International Symposium on Augmented Reality (ISAR 2000), pp. 5 - 6, Oct. 2000

[15] Joan I. Dickinson Ph.D., John P. Marsden Ph.D., Marilyn A. Read Ph.D., "Empirical Design Research: Student Definitions, Perceptions, and Values," Journal of interior Design, vol. 32, no. 2, pp. 1-12, Jan. 2007.

[16] Menk, C., Jundt, E., \& Koch, R., "Visualisation Techniques for Using Spatial Augmented Reality in the Design Process of a Car", Computer Graphics Forum, vol. 30, article. 8, pp. 2354-2366, Oct. 2011.

[17] Nee, A. Y. C., Ong, S. K., Chryssolouris, G., \& Mourtzis, D., "Augmented reality applications in design and manufacturing", CIRP Annals, vol. 61, no. 2, pp. 657-679, 2012.

[18] Jundt E., Menk C., Koch R., "Evaluation of geometric registration methods for using spatial augmented reality in the automotive industry", In 15th International Workshop on Vision, Modeling and Visualization, 2010.

[19] Meola, A., Cutolo, F., Carbone, M., Cagnazzo, F., Ferrari, M. and Ferrari, V., "Augmented reality in neurosurgery: a systematic review," Neurosurgical review, vol. 40, pp.537-548, 2017

[20] Palmarini, R., Erkoyuncu, J.A., Roy, R. and Torabmostaedi, H., "A systematic review of augmented reality applications in maintenance," Robotics and Computer-Integrated Manufacturing, vol. 49, pp.215-228, 2018.

[21] Dacko, S.G., "Enabling smart retail settings via mobile augmented reality shopping apps", Technological Forecasting and Social Change, vol. 124, pp.243-256, 2017.

[22] E. Marchand, H. Uchiyama and F. Spindler, "Pose Estimation for Augmented Reality: A Hands-On Survey," in IEEE Transactions on Visualization and Computer Graphics, vol. 22, no. 12, pp. 2633-2651, 2016.

[23] Wang, Y., Zhang, S., Wan, B., He, W. and Bai, X., "Point cloud and visual feature-based tracking method for an augmented reality-aided mechanical assembly system", The International Journal of Advanced Manufacturing Technology, vol. 99, pp.2341-2352, 2018.

[24] Jain, P., Manweiler, J. and Roy Choudhury, R., "Overlay: Practical mobile augmented reality", In Proceedings of the 13th Annual International Conference on Mobile Systems, Applications, and Services, pp. 331-344, 2015.

[25] Maurer, T., Cook, K. and Graybeal, "Counter-mine augmented reality training system (CMARTS)", In Detection and Sensing of Mines, Explosive Objects, and Obscured Targets XXIV International Society for Optics and Photonics. vol. 11012, p. 1101210, 2019.

[26] Ahmed, et al., "Augmented reality with Haptic technology based online experimental based distance learning education technique," In AIP Conference Proceedings, vol. 1775, no. 1, p. 030068, AIP Publishing, 2016. 